\title{
Quantitative Comparison of Phase Contrast Imaging in Conventional TEM Focal Series and STEM Ptychography
}

\author{
Emanuela Liberti ${ }^{1}$, Hao Yang $^{2}$, Gerardo Martinez ${ }^{1}$, Peter Nellist ${ }^{1}$ and Angus Kirkland ${ }^{1}$ \\ ${ }^{1 .}$ University of Oxford, Department of Materials, Parks Rd, Oxford, UK \\ 2. Lawrence Berkeley National Laboratory, National Center for Electron Microscopy, Molecular \\ Foundry, 1 Cyclotron Rd, Berkeley, CA, USA
}

In phase contrast imaging, three-dimensional, quantitative information about the specimen is encoded in the object wave function, which results from the scattering of the electron wave with the specimen potential. For weak scattering materials, such as a mono-atomic layer of graphene, the phase of the object wave contains all the structural information. However, this phase information is lost in the image recording process. In order to recover the phase, a variety of numerical reconstruction methods are available, including off-axis electron holography [1], focal series reconstruction (FSR) [2] and ptychography [3].

To understand quantitatively materials' properties, matching of experimental phases to simulations is required. In practice, the quantitative information that is obtained from the experimental object wave is often in disagreement with simulations, even for the simple case of a mono-atomic layer of graphene. This disagreement is a phase mismatch, that resembles the contrast mismatch, or Stobbs factor, [4] found between images and simulations.

In this contribution, we focus on a comparison between conventional focal series phase restoration in transmission electron microscopy (TEM) and ptychographic phase restoration in scanning transmission electron microscopy (STEM) for the simple case of graphene. These techniques provide two independent measurements of the phase of a monolayer, which we subsequently compare to explore the physical meaning of the restored object wave phase.

Figure 1 shows the restored phase from a conventional focal series reconstruction (a) and from a ptychography reconstruction (b). The focal series was recorded using an aberration corrected JEOL 2200MCO equipped with an in-column Omega-type energy filter, while the ptychography data set was acquired in a probe corrected JEOL ARM200CF fitted with a direct electron pixelated detector from PNDetector. The detector has an array of $264 \times 264$ pixels and can achieve a speed of up to 20,000 fps through binning/windowing. The focal series and ptycographic reconstructions are based on Wiener filter [5] and Wigner distribution deconvolution [6] algorithms, respectively. Following both restorations, the range of recovered phases is compared for the two methods. The theoretical phase of the object function is also determined for both cases by performing multislice frozen phonon calculations and reproducing, step by step the experimental restoration procedures. The preliminary results of these calculations are shown in Figure 2 for the TEM case. Without introducing any unknown fitting parameters in the simulations, the mismatch between the calculated and experimental phases is found to be 1.7 .

The experimental comparison with the ptycographic simulations, and those for FSR recorded at elevated temperatures, will be further discussed to show the influence of thermal motion on the restored object 
wave, as well as the effects of using zero-loss filtered images to exclude inelastic scattering contributions [7-8].

References:

[1] M. Lehmann et al., Ultramicroscopy 54 (1994) 335 - 344.

[2] W. M. J. Coene et al., Ultramicroscopy 64 (1996) 109 - 135.

[3] J. M. Rodenburg et al., Ultramicroscopy 48 (1993) $304-314$.

[4] M. J. Hÿtch and W.M. Stobbs, Ultramicroscopy 53 (1994) 191 - 203.

[5] A. I. Kirkland et al. Ultramicroscopy 57 (1995) $355-374$.

[6] J. M. Rodenburg and R. H. T. Bates, Philosophical Transactions of the Royal Society A: Mathematical, Physical and Engineering Sciences, 339 (1992) 521-553.

[7] A. Howie, Ultramicroscopy 98 (2004) 73 - 79.

[8] The authors acknowledge funding from the European Union Seventh Framework Programme under Grant Agreement 312483-ESTEEM2 (Integrated Infrastructure Initiative-I3) and from the EPSRC under grant number EP/M010708/1.

(a) FSR Phase (TEM)

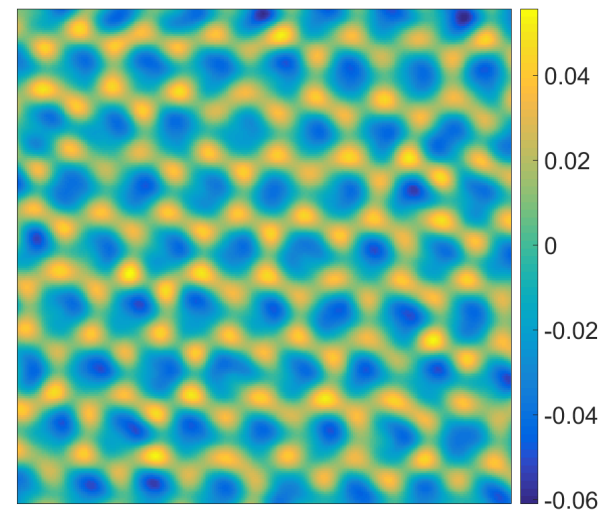

(b) Ptycographic Phase (STEM)

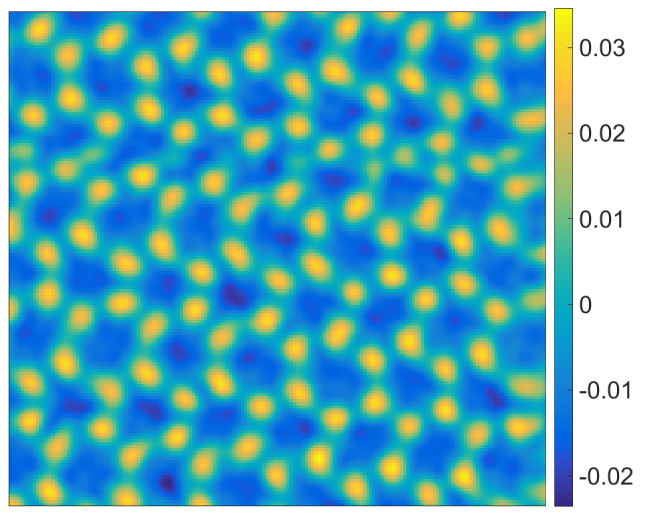

Figure 1. Phase of exit wave function of graphene restored from (a) a conventional focal series of phase contrast TEM images and (b) a ptycographic data set in STEM mode.

(a) Calculated TEM Phase

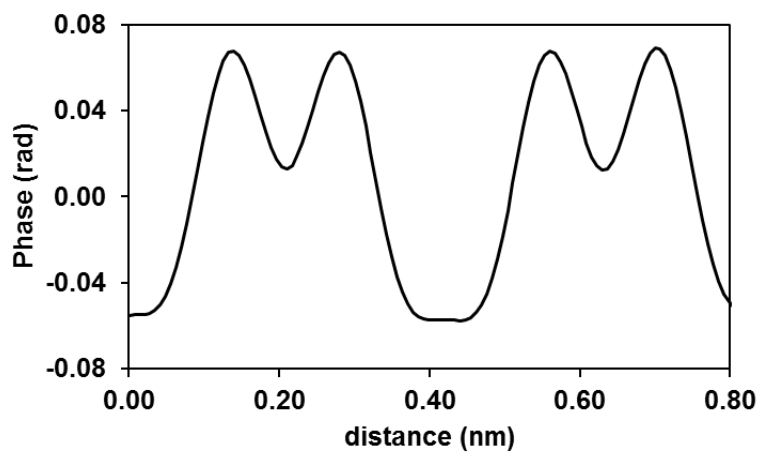

(b) Experimental TEM Phase

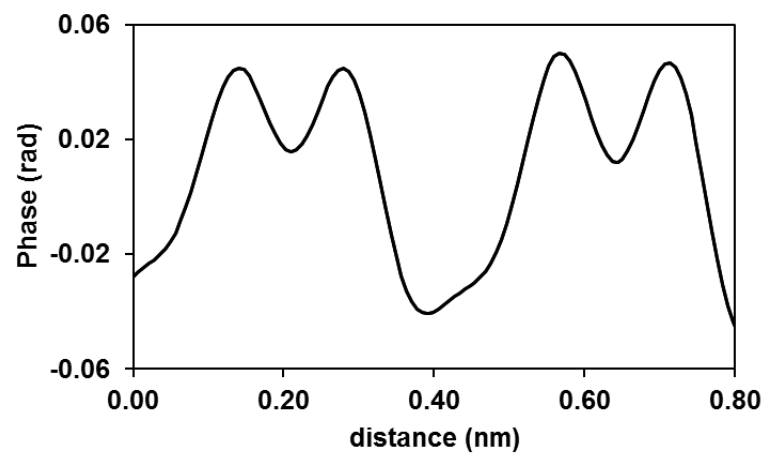

Figure 2. Comparison between (a) calculated and (b) experimental phase profiles of mono-layer graphene for FSR. The phase mismatch between maxima is found to be 1.7. 